\title{
DESIGN, DYNAMIC MODIFICATION, AND ADAPTIVE CONTROL OF A NEW BIPED WALKING ROBOT
}

\author{
AHMAD BAGHERI \\ Department of Mechanical Engineering, Guilan University, \\ Rasht, P.O. Box 3756, Iran \\ bagheri@guilan.ac.ir \\ FARID NAJAFI \\ Faculty of Mechanical Engineering, Khajeh Nassir University, \\ Tehran, Iran \\ fnajafi@kntu.ac.ir \\ REZA FARROKHI \\ Department of Mechanical Engineering, Guilan University, Iran \\ RAHMAN YOUSEFI MOGHADDAM \\ Department of Mechanical and Manufacturing Engineering, \\ University of Manitoba, Winnipeg, Canada \\ umyousef@cc.umanitoba.ca \\ MOHAMMAD EBRAHIM FELEZI \\ Department of Mechanical Engineering, Guilan University, Iran \\ Received 11 January 2005 \\ Revised 12 March 2005
}

\begin{abstract}
Recently, a lot of research has been conducted in the area of biped walking robots that could be compared to human beings. The aim of this article is to control a new planar biped robot by means of an adaptive procedure. The newly designed robot is able to move on its heel like a human. After derivation of dynamic equations of motion for two states of the robot, namely, "supporting leg and trunk" and "swing leg" separately, the stability of robot is achieved by locating the zero moment point (ZMP). A dynamic modification is developed for ZMP positioning. For motion control of the robot, the physical parameters (such as mass, link length and geometry) are estimated (identified) by adaptive methods. A Matlab based software simulation is also conducted.
\end{abstract}

Keywords: Biped walking robot; motion simulation; zero moment point (ZMP); dynamic modification; adaptive control; virtual modeling.

\section{Introduction}

The design, analysis, and fabrication of biped walking robots are the recent interests of many researchers. The kinematics and dynamics modeling of robot, the selection 
of an appropriate control algorithm, walking quality, and stability analysis for such robots are very important problems in this area. Vukobratovic has done pioneering research into motion and control of walking robots. ${ }^{1}$ McGeer has also presented a new passive model for walking mechanisms that moves down an inclined surface without any need for an external actuator or control algorithm. Such a model is set to work on a declined surface according to the robot's gravity. ${ }^{2}$ Also, there are a set of fabricated robots in Waseda university (1969-1999) in which the motion of joints is previously determined. ${ }^{3}$ The $\mathrm{P} 1$ and $\mathrm{P} 2$ are two prototypes that are manufactured at the Honda research center (from 1986 till now). Their movement and control have been done by imitating the joint curve, that is, obtained directly from human body measurements. ${ }^{4}$

\section{The 5 DOF Plane Walking Robot}

The designed plane robot is shown in Fig. 1(a). The considered robot as most the walking robots consists of two sections: upper body and lower body. The motion and the walk of the robot is the duty of lower body and torso is used only for equilibrium. The robot has five degrees of freedom, one of which is in the torso. The lower body part comprises the left and right leg sets.

There are two degrees of freedom in each leg set (four DOF in total). Each leg set consists of the sole, leg and thigh with two parallel mechanisms. Applying the parallel mechanism in the robot results in the inactivity of one of its DOF regardless of the movement limitations. Also, the two robot soles are always parallel and since one of the robot soles is in contact with the ground, the other leg is parallel to the floor.

The mechanism of the sole is designed to simulate and perform the motion of the toe and heel without any increase in the robot's DOF. The sole and its connection to the leg are shown in Fig. 1(b) schematically. Element 1 is the sole and element 3 is the leg, which is circular at the end and in contact with element 1 . The contact

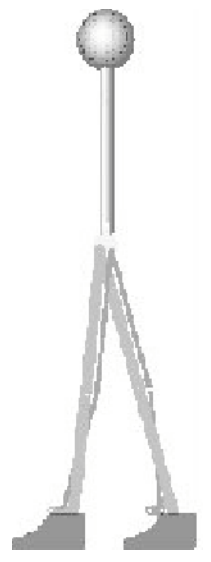

(a)

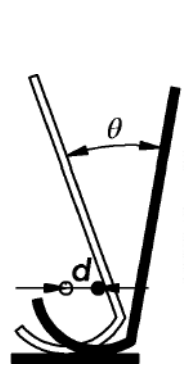

(b)

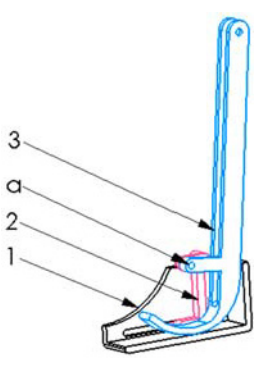

(c)

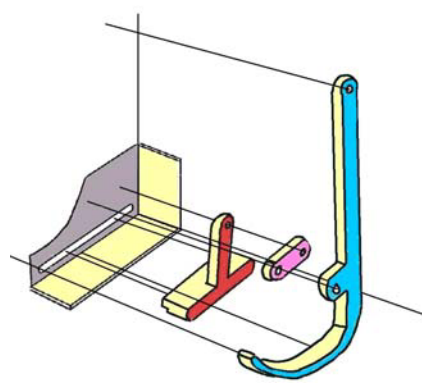

(d)

Fig. 1. Designed robot: (a) plane biped robot; (b) foot and shin model; (c) sole mechanism components; (d) sole mechanism in detail. 
between these two elements ( 1 and 3 ) is by a pinion mechanism. Element 2 is a connecting element which connects elements 1 and 3 . The displacement $d$ of element 2 forces element 3 to translate with respect to element 1, by the amount $d$, and rotate around point "a" shown in Fig. 1(c), by the amount $\theta$. Therefore,

$$
d=R \theta .
$$

The components of the sole mechanism are shown in Fig. 1(c). The mechanism is shown in Fig. 1(d) in detail. Clearly, the additional link, is considered to create the horizontal motion of point "a" by magnitude $d$.

\section{Kinematics of the Robot}

Defining a suitable movement pattern is necessary for the motion of a biped robot. Based on the movement pattern, the proper curve for the assigned parameters of the revolute joints is determined (according to the kinematics relations) and used in the robot's control.

\subsection{Kinematics relations}

A mathematical model of the robot is shown in Figs. 2(a)-(c), which represent the revolute joint variables and geometric parameters, respectively. ( $L_{i}$ is length of the $i$ th link; $b_{i}$ is the center of mass of each component.) As mentioned before, each lower body part, has four degrees of freedom, which according to figures are: $\theta_{1 \mathrm{~L}}, \theta_{2 \mathrm{~L}}, \theta_{1 \mathrm{R}}, \theta_{2 \mathrm{R}}(\mathrm{R}$ and $\mathrm{L}$ are respectively denote right and left legs $)$. The $\left\{0_{\mathrm{L}}\right\}$ coordinate system can be assumed as the reference coordinate system attached to the left leg, and does not have any motion through this step. The left leg is assumed as the supporting leg.

Simplifying the geometric relations, the position of the back leg's sole $\left(\left\{0_{\mathrm{R}}\right\}\right.$ coordinate system) relative to the front leg's sole $\left(\left\{0_{\mathrm{L}}\right\}\right.$ coordinate system), is obtained

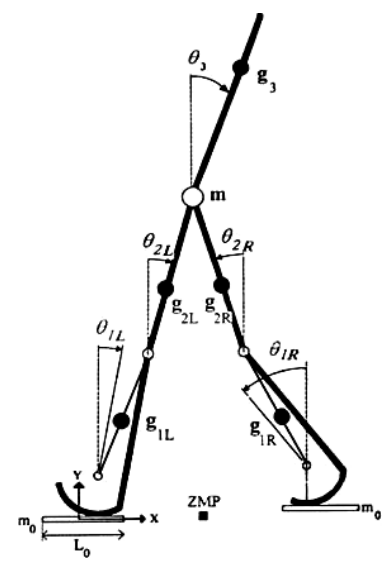

(a)

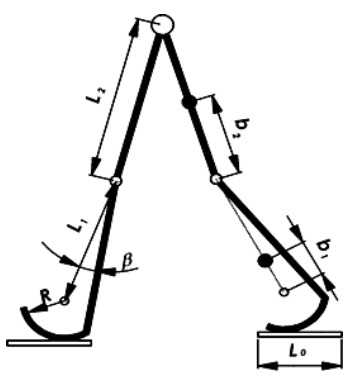

(b)

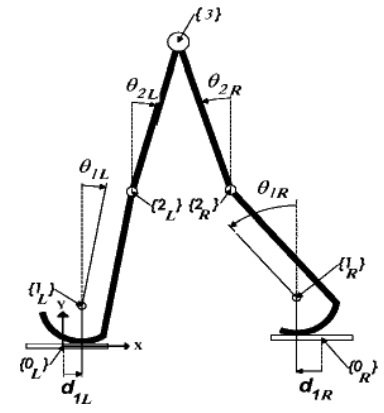

(c)

Fig. 2. Biped robot mathematical model: (a) biped model and ZMP; (b) revolute joint variables; (c) geometrical parameters. 
as the following ${ }^{5}$ :

$$
\begin{aligned}
x_{0 \mathrm{R}}= & d_{1 \mathrm{~L}}+L_{1} \sin \left(\beta+\theta_{1 \mathrm{~L}}\right)+L_{2} \sin \left(\theta_{2 \mathrm{~L}}\right) \\
& -L_{2} \sin \left(\theta_{2 \mathrm{R}}\right)-L_{1} \sin \left(\beta+\theta_{1 \mathrm{R}}\right)-d_{1 \mathrm{R}}, \\
y_{0 \mathrm{R}}= & R+L_{1} \cos \left(\beta+\theta_{1 \mathrm{~L}}\right)+L_{2} \cos \left(\theta_{2 \mathrm{~L}}\right) \\
& -L_{2} \cos \left(\theta_{2 \mathrm{R}}\right)-L_{1} \cos \left(\beta+\theta_{1 \mathrm{R}}\right)-R .
\end{aligned}
$$

In the next section, after defining the movement pattern and solving the equations, different values of revolute joint variables $\left(\theta_{1 \mathrm{~L}}, \theta_{2 \mathrm{~L}}, \theta_{2 \mathrm{R}}, \theta_{1 \mathrm{R}}\right)$ are given for two movement patterns over single and two legs for one of the robot's walking steps. (Note that $d_{1 \mathrm{~L}}, d_{1 \mathrm{R}}$ are not independent variables and, according to the relation (1), are coefficients of $\theta_{1 \mathrm{R}}$ and $\theta_{1 \mathrm{~L}}$.)

\subsection{Movement pattern}

According to the periodic walking process, the determination of the movement pattern for one step is adequate. Similar to the human walk, the robot movement in one step follows two stages: movement "over one leg" and "over two legs."

In the movement over two legs, both of the legs are positioned on the ground. This stage begins by touching the ground with the front leg and ends by separating the back leg from the ground. The beginning and the end of the movement are considered as follows:

(i) At the beginning of the movement, both of the robot knees are straight: $\left(\theta_{2 \mathrm{~L}}=\right.$ $\theta_{1 L}$ and $\left.\theta_{2 \mathrm{R}}=\theta_{1 \mathrm{R}}\right)$.

(ii) At the end of the movement, the front leg's knee (supporting leg) is still straight, but the back leg's knee is bent at the proper angle of $\alpha$. (The value of $\alpha$ is assumed to be 14 degrees $)^{5}: \theta_{2 \mathrm{R}}=\theta_{1 \mathrm{R}}-\alpha$.

In the second stage (movement with one leg), the robot's supporting leg is in contact with the ground, and the oscillating leg separates from the ground, moves from behind the supporting leg, and positions in front of it. The beginning of the movement for each leg is equal to the end of the movement over the two prior legs, and the end of the movement is equal to the beginning of the movement over the two subsequent legs.

Hence,

(i) At the beginning of the movement, the supporting leg's knee is straight and the oscillating leg's knee is bent at as $\alpha$ degrees.

(ii) At the end of the motion, both of the knees are straight.

(iii) During movement, the supporting leg's knee is always straight and the robot separates it from the ground by over-bending the oscillating leg's knee, and moving it in front of the supporting leg.

According to the movement pattern, the kinematic equations are solved for the boundary points, and the curves of the revolute joint variables are derived. ${ }^{6} \mathrm{~A}$ 

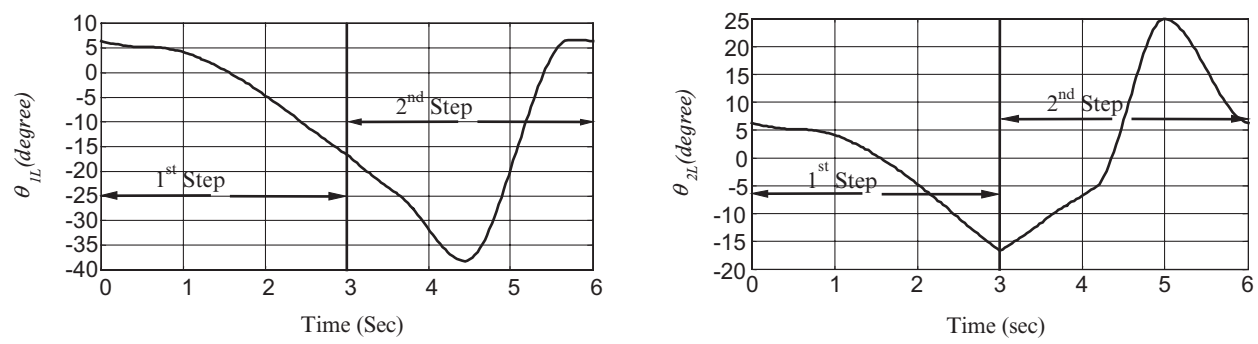

(a) Left leg.
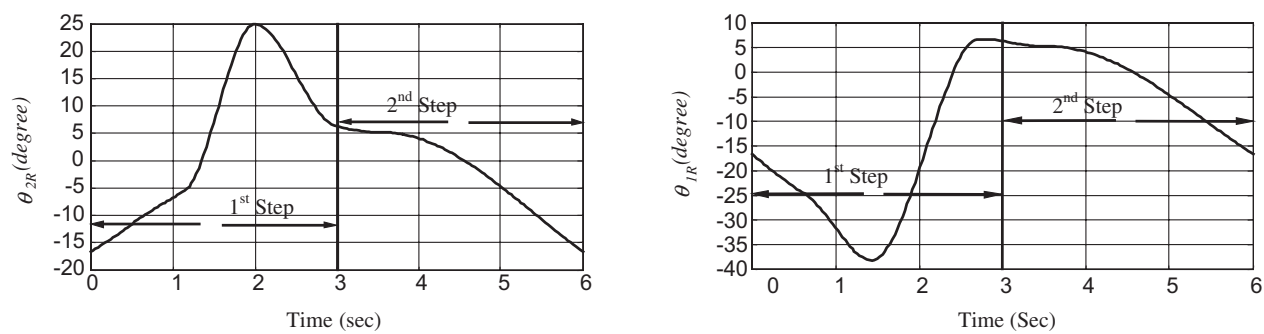

(b) Right leg.

Fig. 3. Lower body revolute joint variables path curve in two steps of the movement.

sample of acceptable results is shown in Fig. 3. According to the periodical motion, as only the place of the oscillating leg and the supporting leg were exchanged, the curves would be the same at the second step.

\section{Modification of the Torso Movement Based on the ZMP Displacement in the Appropriate Points}

Based on the definition, the Zero Moment Point is a point on the datum (ground) that is the result of effective torques, around which the moment is zero, and the exerted reaction force from the earth on the robot is applied to this point. ${ }^{7}$

In the biped robot, the precise position of the zero moment point is determined using the following relation:

$$
x_{\mathrm{ZMP}}=\frac{\sum\left(y_{g i} F_{i x}-x_{g i} F_{i y}\right)+\sum M_{i z}}{\sum F_{i y}},
$$

where $F_{i}=\left[F_{i x}, F_{i y}\right]$ and $M_{i}=\left[M_{i z}\right]$ are the effective forces and the inertial torques on each of the robot links, and calculated by the Newton-Euler equations as in the following:

$$
\begin{aligned}
\vec{F} & =-m \vec{r}_{g}, \\
M_{i} & =-I_{i} \dot{\omega}_{i}-\omega_{i} \times I_{i} \omega_{i} .
\end{aligned}
$$


The vector $r_{g i}=\left[X_{g i}, Y_{g i}\right]$ (and its derivatives) is the position of the mass center (velocity and acceleration) of each component relative to the reference coordinate system $(X Y)$. The path curve of $\theta_{1 \mathrm{~L}}, \theta_{2 \mathrm{~L}}, \theta_{1 \mathrm{R}}, \theta_{2 \mathrm{R}}$ is determined by neglecting the robot balance and stability, and is based on the kinematics equations and the movement pattern (Fig. 3).

Therefore, the torso movement $\left(\theta_{3}\right)$ should be modified in a way that confirms the position of the zero moment point inside an allowable range. For different movement situations, such ranges are as in the following:

(i) Movement over one leg: In this stage, the zero moment point projection always has to be in the polygonal of the supporting leg's sole.

(ii) Movement over two legs: In this stage, the zero moment point allowable range is expanded, and includes the polygonal of both legs' soles.

The proposed zero moment point displacement, during the movement, is shown in Fig. 4(a). ${ }^{8}$ Knowing the $\dot{\theta}_{3}, \theta_{3}$ values at the beginning of the movement, the zero moment point equation is solved and the torso movement $\left(\ddot{\theta}_{3}, \dot{\theta}_{3}, \theta_{3}\right)$ is determined for all paths during the movement. Also, according to the periodical walking process, those curves are acceptable that hold their continuity in the next steps. In other words, the start and the end points and also the tangent of both of points should be equal.

In order to satisfy this specification, the process is as follows:

(i) The movement over one leg: The torso curve, using the assumed zero moment point position Fig. 4(a), and the zero moment point equation solution [relation (4)] are obtained.

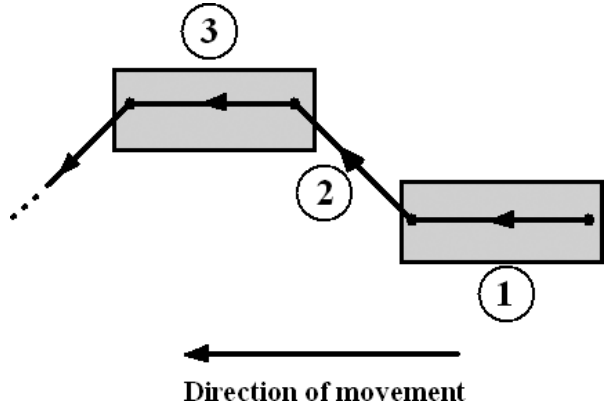

(a) The assumed Zero Moment Point.

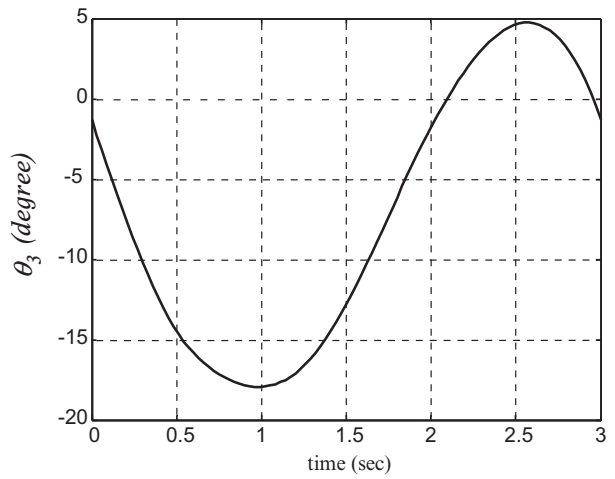

(b) The desired torso curve in the motion over a horizontal surface.

Fig. 4. Torso motion modification based on Zero Moment Point displacement. 


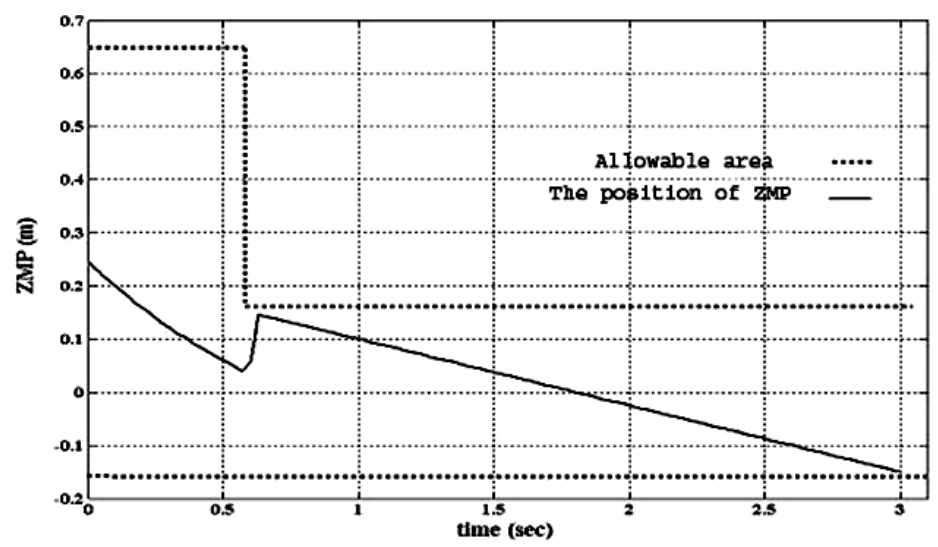

(c) Zero Moment Point inside the allowable range.

Fig. 4. (Continued)

(ii) The movement over two legs: Instead of using the zero moment point equation, a three order curve between the start and end points is fitted and after this, the zero moment point has been controlled and does not exceed the allowable range.

The desired curve of the torso movement is shown in Fig. 4(b). As can be seen in Fig. 4(c), the zero moment point is always in the allowable range, and the robot balance is held.

\section{The Dynamic Equations of the Movement}

The general form of dynamic equations is:

$$
\tau=H(q) \ddot{q}+C(q, \dot{q}) \dot{q}+G(q),
$$

where $q=\left[q_{1}, q_{2}, \ldots, q_{n}\right]$ and $\tau$ are revolute joint variables and applied torques (forces) vector to joints, respectively. In addition, $H, C$ and $G$ are appropriate dynamic matrices. One of the methods for extracting the movement kinematics equations is the Lagrangian dynamic method. ${ }^{9}$ Firstly, based on this method, using the kinetic equations and its derivations, the velocity of the mass center for each component is determined, and each component's potential and kinetic energy are calculated as follows:

$$
\begin{aligned}
T_{i} & =\frac{1}{2} m_{i} V_{g i}^{2}+\frac{1}{2} I_{i} \omega_{i}^{2}, \\
P_{i} & =m_{i} g y_{g i},
\end{aligned}
$$

where $V_{g i}$ is the velocity of the mass center and $\omega_{i}$ is the angular velocity for each component, and $m_{i}, I_{i}$ are the mass and moment inertial of the $i$ th link. $g$ is the 
gravitational acceleration and $y_{g i}$ is of the mass center of the $i$ th link position relative to the reference coordinate system. The total kinetic energy relation is written as follows:

$$
T=\frac{\dot{q}^{T} H \dot{q}}{2} .
$$

Using Eq. (10), the $H(q)$ matrix, is symmetric and positive, is obtained. As mentioned, $C$ and $G$ matrices are determined with the aid of Lagrangian dynamic relations. Defining Lagrangian function $(L=T-P)$, the Lagrangian dynamic equation is

$$
\frac{d}{d t}\left(\frac{\partial L}{\partial \dot{q}}\right)-\frac{\partial L}{\partial q}=\tau
$$

Considering Eq. (10), Eq. (11) is rewritten as

$$
H \ddot{q}+\left(\dot{H} \dot{q}-\frac{\partial T}{\partial q}\right)+\frac{\partial P}{\partial q}=\tau .
$$

Comparing these relations with the general form of the dynamic equation [relation (7)], it can be written as

$$
\begin{aligned}
C \dot{q} & =\dot{H} \dot{q}-\frac{\partial T}{\partial q}, \\
G & =\frac{\partial P}{\partial q} .
\end{aligned}
$$

Thus, calculating the $H(q)$ matrix from relation (10), $C$ and $G$ matrices are obtained from relations (13) and (14).

As mentioned in prior sections, at each stage, two phases of the movement can be assumed: movement phase over one leg and over two legs. As the robot modes are different in these two stages - in the movement over two legs, both of them are in touch with the ground and assumed to be supporting while over the single leg, only one leg is in touch with the ground and the other one is suspended in space - the dynamics of the robot is different and is developed into the two following sequences.

\subsection{Movement over single leg}

Neglecting the effect of the oscillating leg and the supporting leg on each other, the model of the movement dynamics for the single leg is divided into simple and independent models (Fig. 5). The first set is the robot excluding the oscillating leg (includes supporting leg and the torso), and the second set includes the oscillating leg. ${ }^{10,11}$ (If the masses of the leg and the sole are negligible in comparison to the torso, the oscillating leg has no effect on the supporting leg. Also, if the first set of the movement has no acceleration, then the first set has no effect on the second 


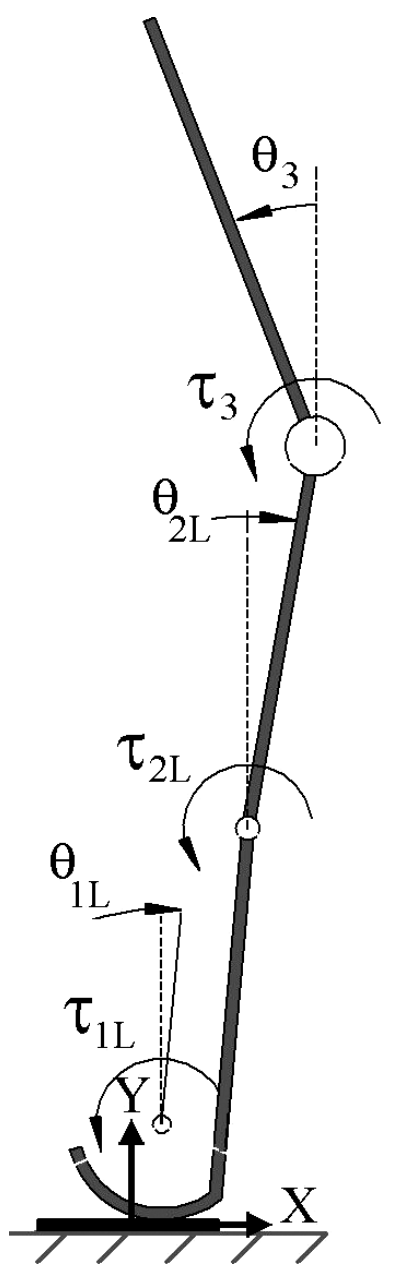

(a) Supporting leg and torso set.

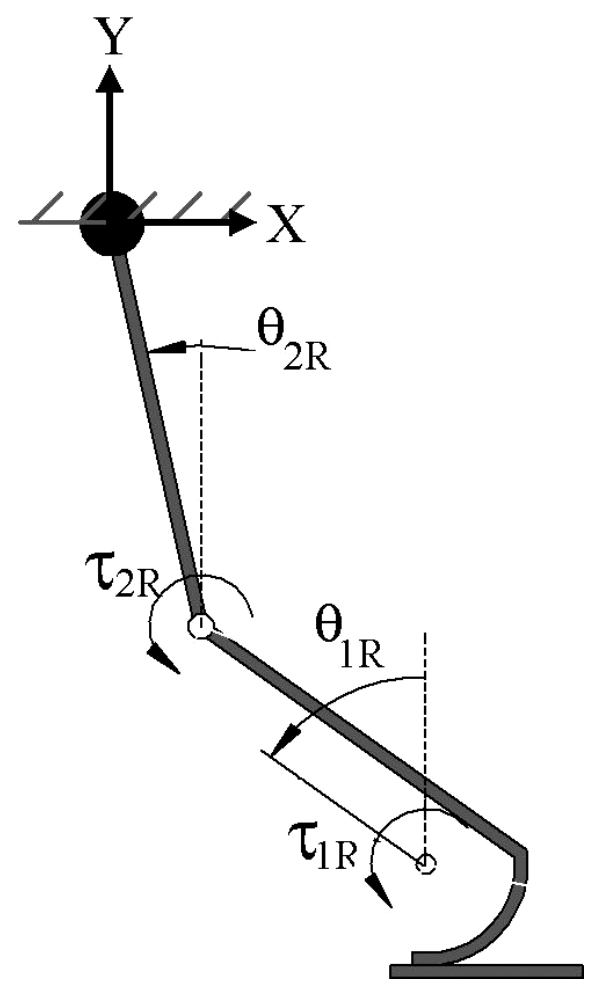

(b) Oscillating leg set.

Fig. 5. Robot division into two sets.

set.) The equation of the movement's dynamics is obtained separately for each of the described sets. Finally, the equation of the movement on a single leg is extracted by compounding the equations.

\subsubsection{The dynamic equations of the supporting leg and torso}

The dynamic model of the supporting leg and the torso is shown in Fig. 5(a). In this model, the robot leg sole (left leg) is assumed to be fixed and the reference coordinate system $(X Y)$ is in the midpoint of the sole. Vector $q$ for this system is $q=\left[\theta_{1 \mathrm{~L}}, \theta_{2 \mathrm{~L}}, \theta_{3 \mathrm{~L}}\right]$. 
Matrices, $H, C$ and $G$ [in Eq. (7)], which is given by the Lagrangian equations) are as follows (L denotes the left leg and su denotes the supporting leg):

$$
\begin{aligned}
& H_{\mathrm{L}}^{\mathrm{SU}}=\left[\begin{array}{ccc}
A_{1}+A_{2} \cos \left(\beta+\theta_{1 \mathrm{~L}}\right) & A_{5} \cos \theta_{2 \mathrm{~L}} & A_{7} \cos \theta_{3} \\
& +A_{6} \cos \left(\beta+\theta_{1 \mathrm{~L}}-\theta_{2 \mathrm{~L}}\right) & +A_{8} \cos \left(\beta+\theta_{1 \mathrm{~L}}-\theta_{3}\right) \\
A_{5} \cos \theta_{2 \mathrm{~L}}+A_{6} \cos \left(\beta+\theta_{1 \mathrm{~L}}-\theta_{2 \mathrm{~L}}\right) & A_{3} & A_{9} \cos \left(\theta_{2 \mathrm{~L}}-\theta_{3}\right) \\
A_{7} \cos \theta_{3}+A_{8} \cos \left(\beta+\theta_{1 \mathrm{~L}}-\theta_{3}\right) & A_{9} \cos \left(\theta_{2 \mathrm{~L}}-\theta_{3}\right) & A_{4}
\end{array}\right], \\
& C_{\mathrm{L}}^{\mathrm{SU}}=\left[\begin{array}{ccc}
-\frac{A_{2}}{2} \sin \left(\beta+\theta_{1 \mathrm{~L}}\right) \dot{\theta}_{1 \mathrm{~L}} & \left(-A_{5} \sin \theta_{2 \mathrm{~L}}\right. & \left(-A_{7} \sin \theta_{3}\right. \\
-A_{5} \sin \theta_{2 \mathrm{~L}} \dot{\theta}_{2 \mathrm{~L}} & \left.A_{6} \sin \left(\beta+\theta_{1 \mathrm{~L}}-\theta_{2 \mathrm{~L}}\right)\right) \dot{\theta}_{2 \mathrm{~L}} \sin \theta_{2 \mathrm{~L}} \dot{\theta}_{1 \mathrm{~L}} & \left.+A_{8} \sin \left(\beta+\theta_{1 \mathrm{~L}}-\theta_{3}\right)\right) \dot{\theta}_{3} \\
-A_{6} \sin \left(\beta+\theta_{1 \mathrm{~L}}-\theta_{2 \mathrm{~L}}\right) \dot{\theta}_{1 \mathrm{~L}} & A_{9} \sin \left(\theta_{2 \mathrm{~L}}-\theta_{3}\right) \dot{\theta}_{3} \\
-A_{7} \dot{\theta}_{3} \sin \theta_{3} & -A_{9} \sin \left(\theta_{2 \mathrm{~L}}-\theta_{3}\right) \dot{\theta}_{2 \mathrm{~L}} & \\
-A_{8} \sin \left(\beta+\theta_{1}-\theta_{3}\right) \dot{\theta}_{1 \mathrm{~L}} & A_{7} \sin \theta_{3} \dot{\theta}_{1 \mathrm{~L}}
\end{array}\right], \\
& G_{\mathrm{L}}^{\mathrm{SU}}=\left[\begin{array}{c}
-\frac{A_{2}}{2 R} g \sin \left(\beta+\theta_{1 \mathrm{~L}}\right) \\
-\frac{A_{5}}{R} g \sin \theta_{2 \mathrm{~L}} \\
-\frac{A_{7}}{R} g \sin \theta_{3}
\end{array}\right] .
\end{aligned}
$$

In the above relations, the terms $A_{1}$ to $A_{9}$ include the physical system parameters and are equal to:

$$
\begin{aligned}
& A_{1}=R^{2} m_{1}+m_{1} b_{1}^{2}+I_{1}+R^{2} m_{2}+m_{2} L_{1}^{2}+R^{2} m+m L_{1}^{2}+R^{2} m_{3}+m_{3} L_{1}^{2}, \\
& A_{2}=2 R\left(m_{1} b_{1}+m_{2} L_{1}+m L_{1}+m_{3} L_{1}\right), \quad A_{3}=m_{2} b_{2}^{2}+I_{2}+m L_{2}^{2}+m_{3} L_{2}^{2}, \\
& A_{4}=m_{3} b_{3}^{2}+I_{3}, \quad A_{5}=R m_{2} b_{2}+R m L_{2}+R m_{3} L_{2}, \\
& A_{6}=m_{2} L_{1} b_{2}+m L_{1} L_{2}+m_{3} L_{1} L_{2}, \quad A_{7}=R m_{3} b_{3}, \\
& A_{8}=m_{3} L_{1} b_{3}, \quad A_{9}=m_{3} L_{2} b_{3} .
\end{aligned}
$$

\subsubsection{The dynamic equations of the oscillating leg}

The dynamic model of an oscillating leg is shown in Fig. 5(b). The position of the reference coordinate system $(X Y)$ is shown also. Vector, $(q)$ is: $q=\left[\theta_{1 \mathrm{R}}, \theta_{2 \mathrm{R}}\right]$.

Matrices, $H, C$ and $G$ (in Eq. (7), which are obtained from the Lagrangian equations) are as follows: (R denotes the right leg, and SW denotes the supporting $\operatorname{leg})$ :

$H_{\mathrm{R}}^{\mathrm{SW}}=\left[\begin{array}{cc}B_{1}+B_{2} \cos \left(\beta+\theta_{1 \mathrm{R}}\right) & B_{4} \cos \left(\beta+\theta_{1 \mathrm{R}}-\theta_{2 \mathrm{R}}\right)+B_{5} \cos \theta_{2 \mathrm{R}} \\ B_{4} \cos \left(\beta+\theta_{1 \mathrm{R}}-\theta_{2 \mathrm{R}}\right)+B_{5} \cos \theta_{2 \mathrm{R}} & B_{3}\end{array}\right]$, 


$$
\begin{aligned}
C_{\mathrm{R}}^{\mathrm{SW}} & =\left[\begin{array}{cc}
-\frac{B_{2}}{2} \sin \left(\beta+\theta_{1 \mathrm{R}}\right) \dot{\theta}_{1 \mathrm{R}} & \left(B_{4} \sin \left(\beta+\theta_{1 \mathrm{R}}-\theta_{2 \mathrm{R}}\right)\right. \\
-B_{4} \sin \left(\beta+\theta_{1 \mathrm{R}}-\theta_{2 \mathrm{R}}\right) \dot{\theta}_{1 \mathrm{R}} & \left.-B_{5} \sin \theta_{2 \mathrm{R}}\right) \dot{\theta}_{2 \mathrm{R}} \\
-B_{5} \sin \theta_{2 \mathrm{R}} \dot{\theta}_{2 \mathrm{R}} & B_{5} \sin \theta_{2 \mathrm{R}} \dot{\theta}_{1 \mathrm{R}}
\end{array}\right], \\
G_{\mathrm{R}}^{\mathrm{SW}} & =\left[\begin{array}{c}
B_{6} g \sin \left(\beta+\theta_{1 \mathrm{R}}\right) \\
B_{7} g \sin \theta_{2 \mathrm{R}}
\end{array}\right],
\end{aligned}
$$

where the terms $B_{1}$ to $B_{7}$ are the system's physical parameters (such as length and mass) and are equal to:

$$
\begin{aligned}
& B_{1}=m_{1}\left(L_{1}-b_{1}\right)^{2}+m_{0}\left(L_{1}^{2}+R^{2}\right)+I_{1}, \quad B_{2}=2 m_{0} R L_{1}, \\
& B_{3}=m_{2}\left(l_{2}-b_{2}\right)^{2}+I_{2}+m_{1} l_{2}^{2}+m_{0} l_{2}^{2}, \quad B_{4}=m_{1}\left(L_{1}-b_{1}\right) L_{2}+m_{0} L_{1} L_{2}, \\
& B_{5}=m_{0} R l_{2}, \quad B_{6}=m_{1}\left(L_{1}-b_{1}\right)+m_{0} L_{1}, \quad B_{7}=m_{2}\left(l_{2}-b_{2}\right)+m_{1} l_{2}+m_{0} l_{2} .
\end{aligned}
$$

Now, compounding matrices, $H, C$ and $G$, obtained for the oscillating leg, the supporting leg and the torso, the dynamic equations of the robot for the movement on a single leg are equal to:

$$
\left[\begin{array}{c}
\tau_{1 \mathrm{~L}} \\
\tau_{2 \mathrm{~L}} \\
\tau_{3} \\
\tau_{1 \mathrm{R}} \\
\tau_{2 \mathrm{R}}
\end{array}\right]=\left[\begin{array}{cc}
H_{\mathrm{L}}^{\mathrm{SU}} & 0 \\
0 & H_{\mathrm{R}}^{\mathrm{SW}}
\end{array}\right]_{5 \times 5}\left[\begin{array}{c}
\ddot{\theta}_{1 \mathrm{~L}} \\
\ddot{\theta}_{2 \mathrm{~L}} \\
\ddot{\theta}_{3} \\
\ddot{\theta}_{1 \mathrm{R}} \\
\ddot{\theta}_{2 \mathrm{R}}
\end{array}\right]+\left[\begin{array}{cc}
C_{\mathrm{L}}^{\mathrm{SU}} & 0 \\
0 & C_{\mathrm{R}}^{\mathrm{SW}}
\end{array}\right]_{5 \times 5}\left[\begin{array}{c}
\dot{\theta}_{1 \mathrm{~L}} \\
\dot{\theta}_{2 \mathrm{~L}} \\
\dot{\theta}_{3} \\
\dot{\theta}_{1 \mathrm{R}} \\
\dot{\theta}_{2 \mathrm{R}}
\end{array}\right]+\left[\begin{array}{c}
G_{\mathrm{L}}^{\mathrm{SU}} \\
G_{\mathrm{R}}^{\mathrm{SW}}
\end{array}\right]_{5 \times 1} .
$$

\subsection{Movement over two legs}

In the movement over two legs, both legs are assumed to be supporting legs and, for dynamic analysis, the effective forces on each leg or the effect of each leg on the robot walk should be determined. The method used in the present paper is the expanded form of the method mentioned in Ref. 11. In this method, the model of the robot (similar to the movement on a single leg) is divided in two separate systems including the supporting leg, the torso and the oscillating leg (Fig. 6). Note that the left leg parameters $\kappa$ and $(1-\kappa)$, are assumed to be the supporting and the oscillating leg, respectively. Therefore, the right leg is the oscillating leg by considering the parameter $\kappa$ and the supporting leg by considering the parameter $(1-\kappa)$.

Parameter $\kappa$ is a parameter for dividing force between each of the two sets shown in Fig. 6 as follows:

$$
\kappa=\frac{x_{\text {right }}}{x_{\text {right }}+x_{\text {left }}},
$$

where $X_{\text {left }}$, and $X_{\text {right }}$ are the horizontal distances of the zero moment point from the left sole and the right sole, respectively. 

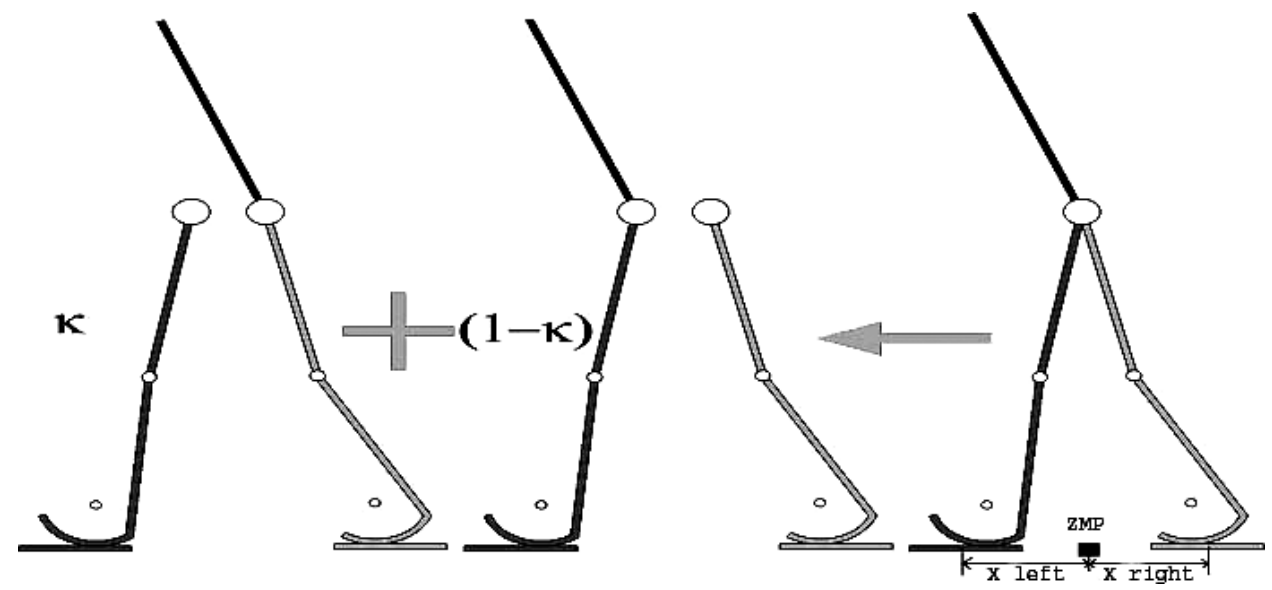

Fig. 6. Movement division between each robot leg in movement over two legs.

This means that if the zero moment point is assumed to be on the right sole, then $\kappa=0$, and if it is on the left leg sole, then $\kappa=1$; for the state between these situations, $\kappa$ will be between zero and unity $(0<\kappa<1)$. Hence, the dynamic equations of the movement over two legs (by considering the parameter $\kappa$ ), is obtained from the following summation of items:

(a) Supporting leg and torso system includes: Left Leg and The Torso. Oscillating leg system includes: Left Leg.

(b) Supporting leg system includes: Right Leg and The Torso. Oscillating leg system includes: Left Leg.

The equations of both the (a) and (b) cases are similar to the equations obtained in the previous section [relation (21)]. Hence, for the movement over two legs, it can be written:

$$
\left[\begin{array}{c}
(a) \\
{\left[\begin{array}{c}
\tau_{1 \mathrm{~L}} \\
\tau_{2 \mathrm{~L}} \\
\tau_{3} \\
\tau_{1 \mathrm{R}} \\
\tau_{2 \mathrm{R}}
\end{array}\right]=\kappa\left[\begin{array}{c}
\tau_{1 \mathrm{~L}} \\
\tau_{2 \mathrm{~L}} \\
\tau_{3} \\
\tau_{1 \mathrm{R}} \\
\tau_{2 \mathrm{R}}
\end{array}\right]+(1-\kappa)\left[\begin{array}{c}
\tau_{1 \mathrm{~L}} \\
\tau_{2 \mathrm{~L}} \\
\tau_{3} \\
\tau_{1 \mathrm{R}} \\
\tau_{2 \mathrm{R}}
\end{array}\right] .}
\end{array}\right.
$$

\section{Adaptive Control}

The adaptive control procedure is a nonlinear control method used in robotics. Assuming the revolute joint curves $q_{d}(t)$ are known and all or some of the robot parameters are unknown, the problem of designing the adaptive controller includes obtaining a control law for revolute joint torques and determining the estimation 
law for unknown parameters, where the robot output $q(t)$ follows the desired curve path precisely. ${ }^{12}$

This method includes two steps as follows:

(i) Physical system parameter estimation (using adaptive control).

(ii) Robot control in a specific path, with the aid of an adaptive law and estimated parameters in the first step.

For controller parameter regulation, a new physical property is defined for the system called the "unknown system parameters vector," $a$, which is the system's geometric and inertial properties indicator. This vector is defined such that different components of the dynamics equations, i.e. $C(q, \dot{q}), H(q)$ matrices and vector $G(q)$, become a linear function of the " $a$ " vector.

With the above definition, matrix $Y\left(q, \dot{q}, \dot{q}_{r}, \ddot{q}_{r}\right)$ can be given in the following form (by rewriting the dynamic equations):

$$
\tau=H(q) \ddot{q}_{r}+C(q, \dot{q}) \dot{q}_{r}+G(q)=Y\left(q, \dot{q}, \dot{q}_{r}, \ddot{q}_{r}\right) a .
$$

In the above relation, $\dot{q}_{r}$ is the velocity vector of the reference and is obtained from the modification of the desired velocity vector $\dot{q}_{d}$ and according to the error vector $\tilde{q}$ as follows:

$$
\dot{q}_{r}=\dot{q}_{d}-\Lambda \tilde{q},
$$

where matrix $\Lambda$ is a positive diagonal matrix. Also, a new vector $s$ is the virtual velocity error vector and is determined from the difference of $\dot{q}_{r}$ and $\dot{q}$ :

$$
s=\dot{q}-\dot{q}_{r} .
$$

Hence, the control law used in adaptive control is given as

$$
\tau=Y \hat{a}-K_{D} \cdot s .
$$

The parameter estimation law that is defined ${ }^{12}$ is the integral of the following relation:

$$
\dot{\hat{a}}=-\Gamma Y^{T} s .
$$

The law is considered such that by substituting it into the Lyapunov function derivative below, its value is always smaller than zero, and then the Lyapunov stability condition is satisfied:

$$
V(t)=\frac{1}{2}\left[s^{T} H s+\tilde{a}^{T} \Gamma^{-1} \tilde{a}\right] .
$$

$K_{D}$ and $\Gamma$ are positive diagonal matrices. The time derivative of the above relation is

$$
\dot{V}(t)=s^{T}\left(H \ddot{q}-H \ddot{q}_{r}\right)+\frac{1}{2} s^{T} \dot{H} s+\dot{\hat{a}}^{T} \Gamma^{-1} \tilde{a} .
$$

Simplifying relation (30), we obtain

$$
\dot{V}(t)=-s^{T} K D s \leq 0 .
$$

As can be seen from relation (31), the derivative of the considered relation (29) is always equal to or smaller than zero and therefore satisfies the Lyapunov stability 
condition. Also, it can be noticed that the state variable values have a proper maximum that do not approach to infinity. ${ }^{13}$

As mentioned, after converging all the elements of the vector $a$ to constant values, the values can be used in system controlling.

Simplifying relation (27), the adaptive control law is given as follows:

$$
\tau=\hat{H} \ddot{q}_{d}+\hat{C} \dot{q}_{d}+\hat{G}-K_{P} \tilde{q}-K_{V} \dot{\tilde{q}}
$$

where the diagonal matrices $K_{p}, K_{v}$ are assumed to be feedback and controller coefficients and are calculated as

$$
\begin{aligned}
& K_{P}=\hat{C}(q, \dot{q}) \Lambda+K_{D} \Lambda, \\
& K_{V}=\hat{H}(q) \Lambda+K_{D} .
\end{aligned}
$$

The adaptive control procedure, which just defined, is shown in Fig. 7(a).

In order to estimate the parameters and to control the robot, computer simulation is used. The simulation method is shown in Fig. 7(b). The dynamic model of the robot is simulated in Visual Nastran Desktop (VND) and the control system is simulated using Matlab software. The new specification of the VND makes it possible to have a connection between two software programs and achieve information transfer.

\subsection{Supporting leg and torso parameters estimation}

The dynamic model of the system is shown in Fig. 5(a). According to the movement dynamic equations, the parameter vector $a$ can be written for this set as

$$
a=\left[\begin{array}{lllllllll}
A_{1} & A_{2} & A_{3} & A_{4} & A_{5} & A_{6} & A_{7} & A_{8} & A_{9}
\end{array}\right]^{T} .
$$

Substituting and simplifying the model matrices $H, C$ and $G$ relations (15)-(17) into relation (24), the elements of the $\left(Y_{3 \times 9}\right)$ matrix are given as follows (for

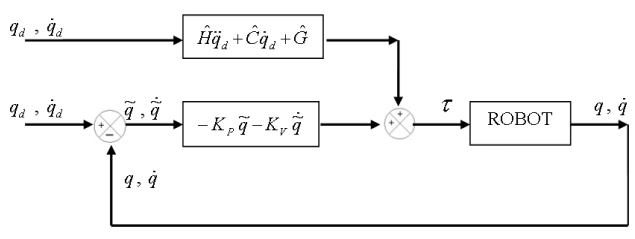

(a) Adaptive control block diagram.

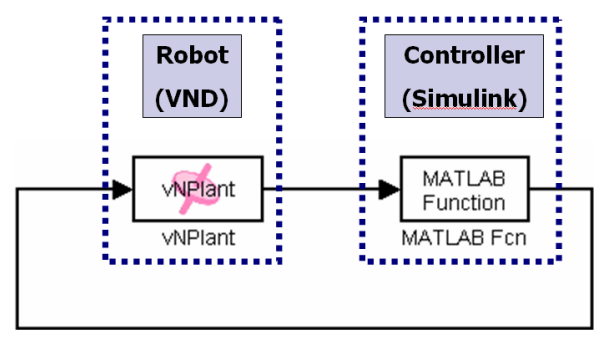

(b) Computer based simulation.

Fig. 7. Biped robot simulation and adaptive control. 
simplicity, subscript L is neglected):

$$
\begin{aligned}
& Y_{11}=\ddot{\theta}_{1 \mathrm{r}}, \quad Y_{13}=0, \quad Y_{14}=0, \quad Y_{19}=0, \\
& Y_{12}=\cos \left(\beta+\theta_{1}\right) \ddot{\theta}_{1 \mathrm{r}}-\frac{1}{2} \sin \left(\beta+\theta_{1}\right) \dot{\theta}_{1} \dot{d}_{1 \mathrm{r}}-\frac{g}{2 R} \sin \left(\beta+\theta_{1}\right), \\
& Y_{15}=\cos \left(\theta_{2}\right) \ddot{\theta}_{2 \mathrm{r}}-\sin \left(\theta_{2}\right) \dot{\theta}_{2} \dot{\theta}_{2 \mathrm{r}}, \\
& Y_{16}=\cos \left(\beta+\theta_{1}-\theta_{2}\right) \ddot{\theta}_{2 \mathrm{r}}+\sin \left(\beta+\theta_{1}-\theta_{2}\right) \dot{\theta}_{2} \dot{\theta}_{2 \mathrm{r}}, \\
& Y_{17}=\cos \left(\theta_{3}\right) \ddot{\theta}_{3 r}-\sin \left(\theta_{3}\right) \dot{\theta}_{3} \dot{\theta}_{3 r}, \\
& Y_{18}=\cos \left(\beta+\theta_{1}-\theta_{3}\right) \ddot{\theta}_{3 r}+\sin \left(\beta+\theta_{1}-\theta_{3}\right) \dot{\theta}_{3} \dot{\theta}_{3 r}, \\
& Y_{21}=Y_{22}=Y_{24}=Y_{27}=Y_{28}=0, \quad Y_{23}=\ddot{\theta}_{2 \mathrm{r}}, \\
& Y_{25}=\cos \left(\theta_{2}\right) \ddot{d}_{1 \mathrm{r}}-\sin \left(\theta_{2}\right) \dot{\theta}_{2} \dot{\theta}_{1 \mathrm{r}}+\sin \left(\theta_{2}\right) \dot{\theta}_{2 \mathrm{r}} \dot{\theta}_{1}-g \sin \left(\theta_{2}\right), \\
& Y_{26}=\cos \left(\beta+\theta_{1}-\theta_{2}\right) \ddot{d}_{1 \mathrm{r}}-\sin \left(\beta+\theta_{1}-\theta_{2}\right) \dot{\theta}_{1} \dot{\theta}_{1 \mathrm{r}}, \\
& Y_{29}=\cos \left(\theta_{2}-\theta_{3}\right) \ddot{\theta}_{3 r}+\sin \left(\theta_{2}-\theta_{3}\right) \dot{\theta}_{3} \dot{\theta}_{3 r}, \\
& Y_{31}=Y_{32}=Y_{33}=Y_{35}=Y_{36}=0, \quad Y_{34}=\ddot{\theta}_{3 r}, \\
& Y_{37}=\cos \left(\theta_{3}\right) \ddot{\theta}_{1 \mathrm{r}}-\sin \left(\theta_{3}\right) \dot{\theta}_{3} \dot{\theta}_{1 \mathrm{r}}+\sin \left(\theta_{3}\right) \dot{\theta}_{3 r} \dot{\theta}_{1}-\frac{g}{R} \sin \left(\theta_{3}\right), \\
& Y_{38}=\cos \left(\beta+\theta_{1}-\theta_{3}\right) \ddot{\theta}_{1 \mathrm{r}}-\sin \left(\beta+\theta_{1}-\theta_{3}\right) \dot{\theta}_{1} \dot{\theta}_{1 \mathrm{r}}, \\
& Y_{39}=\cos \left(\theta_{2}-\theta_{3}\right) \ddot{\theta}_{2 \mathrm{r}}-\sin \left(\theta_{2}-\theta_{3}\right) \dot{\theta}_{2} \dot{\theta}_{2 \mathrm{r}} .
\end{aligned}
$$

The model's revolute joint variables are $\theta_{1}, \theta_{2}, \theta_{3}$ and the desired revolute joint curves $\left(q_{d}(t)\right)$, which are assumed to be periodic functions (estimated), are

$$
\begin{aligned}
& \theta_{1 d}=0.033 \cos (\pi t)+0.066 \\
& \theta_{2 d}=0.05 \cos (\pi t)+0.21+0.005 \cos (2 \pi t)-0.005, \\
& \theta_{3 d}=-0.3 \cos (\pi t)-0.15+0.003 \cos (5 \pi t)-0.003 .
\end{aligned}
$$

The matrices used $K_{D}, \Gamma, \Lambda$ for this model are

$$
\begin{aligned}
K_{D} & =\left[\begin{array}{rrr}
35 & 0 & 0 \\
0 & 20 & 0 \\
0 & 0 & 40
\end{array}\right], \quad \Lambda=\left[\begin{array}{rrr}
30 & 0 & 0 \\
0 & 30 & 0 \\
0 & 0 & 30
\end{array}\right], \\
\Gamma & =\operatorname{diag}[0.4,0.1,1.1,0.2,0.1,0.8,0.08,0.3,0.6] .
\end{aligned}
$$

After running the computer simulation for 350 seconds all physical parameters of the system, converge to constant values. The final magnitudes of those parameters are

$$
\begin{gathered}
\hat{A}_{1}=0.98, \quad A_{2}=2.25, \quad \hat{A}_{3}=5.4, \quad \hat{A}_{4}=-0.54, \quad \hat{A}_{5}=1.16, \\
\hat{A}_{6}=3.1, \quad \hat{A}_{7}=1.2, \quad \hat{A}_{8}=-0.7, \quad \hat{A}_{9}=0.98 .
\end{gathered}
$$

For instance, the convergence of $A_{1}, A_{5}$ and $A_{9}$ parameters are shown in Fig. 8. 

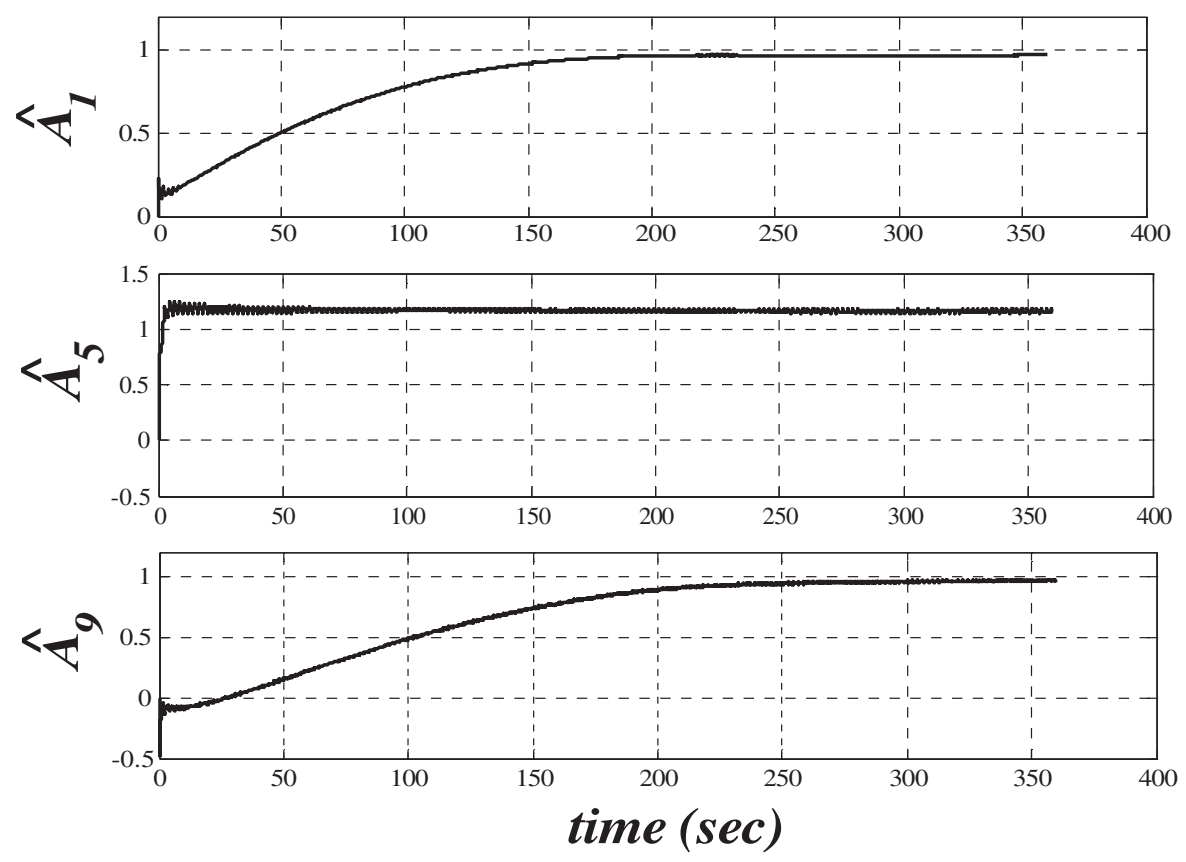

Fig. 8. Estimation of the torso and supporting leg parameters.

\subsection{Oscillating leg set parameters estimation}

The dynamic model of this set is shown in Fig. 5(b). According to the dynamic equations of the movement, the parameter vector $a$ is given as

$$
a=\left[\begin{array}{lllllll}
B_{1} & B_{2} & B_{3} & B_{4} & B_{5} & B_{6} & B_{7}
\end{array}\right]^{T} .
$$

Substituting the model matrices $H, C$ and $G$ [relations (18)-(20)] into relation (24) and simplifying, the elements of the $Y_{2 \times 7}$ matrix are obtained as follows (for simplicity, subscript $\mathrm{R}$ is neglected):

$$
\begin{aligned}
& Y_{11}=\ddot{\theta}_{1 \mathrm{r}}, \quad Y_{16}=g \sin \left(\beta+\theta_{1}\right), \quad Y_{13}=Y_{17}=0, \\
& Y_{12}=\cos \left(\beta+\theta_{1}\right) \ddot{\theta}_{1 \mathrm{r}}-\frac{1}{2} \sin \left(\beta+\theta_{1}\right) \dot{\theta}_{1} \dot{\theta}_{1 \mathrm{r}}, \\
& Y_{14}=\cos \left(\beta+\theta_{1}-\theta_{2}\right) \ddot{\theta}_{2 \mathrm{r}}+\sin \left(\beta+\theta_{1}-\theta_{2}\right) \dot{\theta}_{2} \dot{\theta}_{2 \mathrm{r}}, \\
& Y_{15}=\cos \left(\theta_{1}\right) \ddot{\theta}_{2 \mathrm{r}}-\sin \left(\theta_{2}\right) \dot{\theta}_{2} \dot{\theta}_{2 \mathrm{r}}, \\
& Y_{21}=Y_{22}=0, \quad Y_{23}=\ddot{\theta}_{2 \mathrm{r}}, \quad Y_{27}=g \sin \left(\theta_{2}\right), \\
& Y_{24}=\cos \left(\beta+\theta_{1}-\theta_{2}\right) \ddot{\theta}_{1 \mathrm{r}}-\sin \left(\beta+\theta_{1}-\theta_{2}\right) \dot{\theta}_{1} \dot{\theta}_{1 \mathrm{r}}, \\
& Y_{15}=\cos \left(\theta_{2}\right) \ddot{\theta}_{1 \mathrm{r}}-\sin \left(\theta_{2}\right) \dot{\theta}_{2} \dot{\theta}_{1 \mathrm{r}}+\sin \left(\theta_{2}\right) \dot{\theta}_{1} \dot{\theta}_{2 \mathrm{r}} .
\end{aligned}
$$


The revolute joint variables of the model are $\theta_{1}, \theta_{2}$ and the desired revolute joint curves $\left(q_{d}(t)\right)$ are assumed as a periodic function parameter estimation:

$$
\begin{aligned}
& \theta_{1 d}=-0.13 \cos (\pi t)-0.53+0.01 \cos (2 \pi t)-0.01 \\
& \theta_{2 d}=-\frac{\pi}{36} \cos (\pi t)-\frac{\pi}{12}+\frac{\pi}{360} \cos (2 \pi t)-\frac{\pi}{360} .
\end{aligned}
$$

Also, the matrices, $K_{D}, \Gamma$ and $\Lambda$, which are used for the model, are given as

$$
\begin{aligned}
K_{D} & =\left[\begin{array}{rr}
15 & 0 \\
0 & 15
\end{array}\right], \quad \Lambda=\left[\begin{array}{rrrr}
40 & 0 \\
0 & 40
\end{array}\right] \\
\Gamma & =\operatorname{diag}\left[\begin{array}{lllllll}
0.1 & 0.1 & 0.4 & 0.1 & 0.1 & 0.02 & 0.02
\end{array}\right] .
\end{aligned}
$$
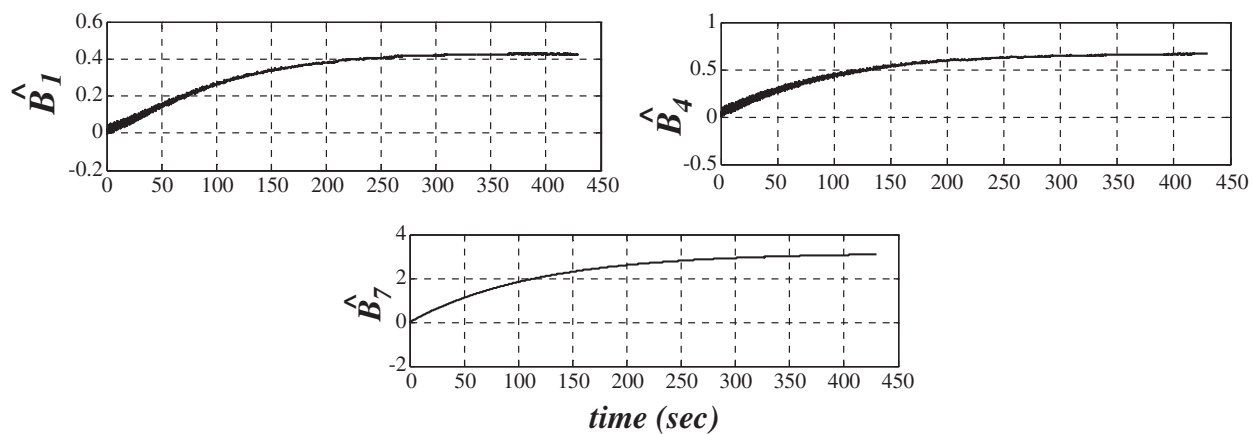

Fig. 9. Oscillating leg parameters estimation.
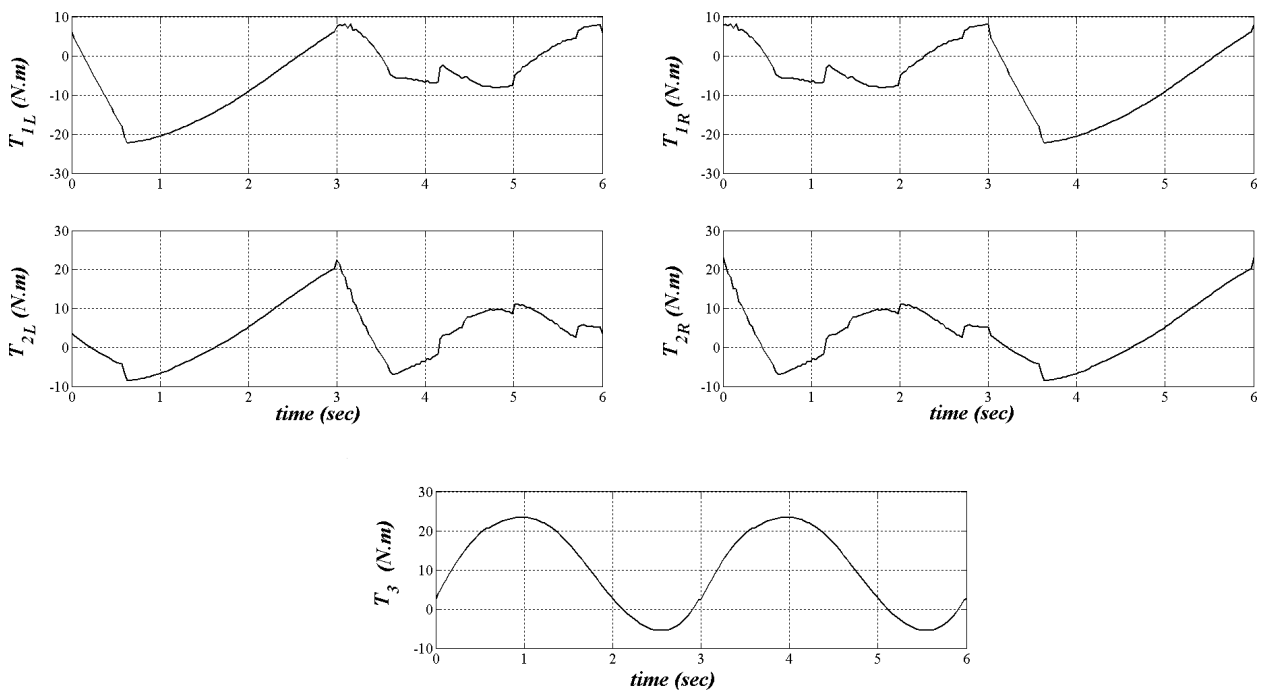

Fig. 10. Revolute joint torque diagrams. 


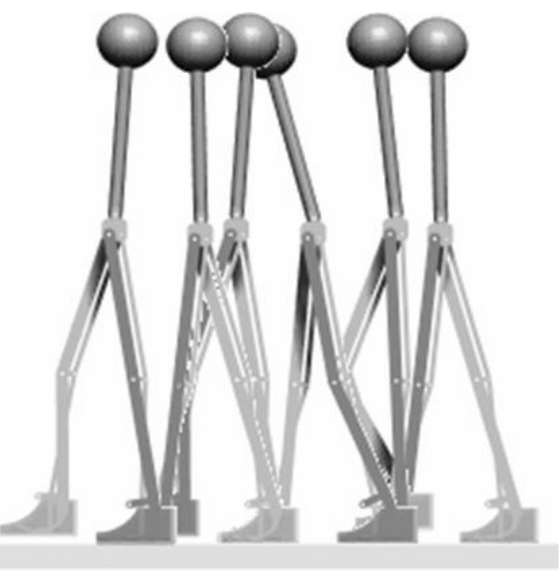

Fig. 11. Computer simulation in VND software.
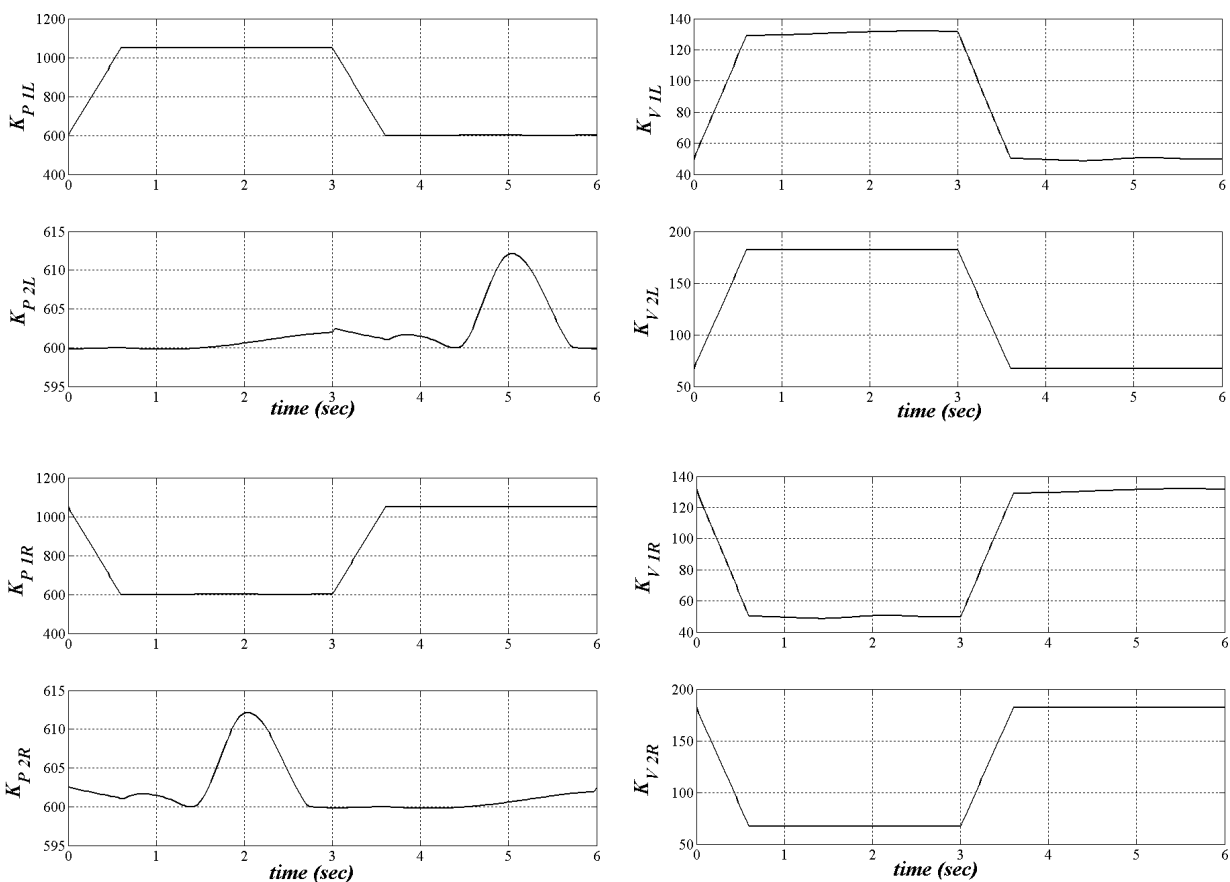

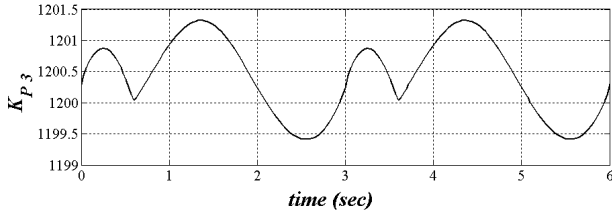

(a) Matrix $K_{p}$

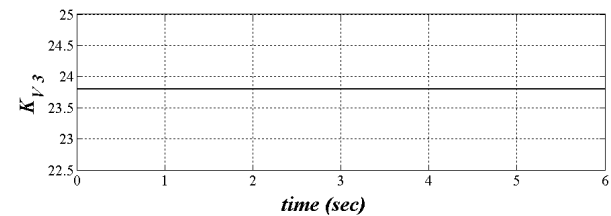

(b) Matrix $K_{v}$

Fig. 12. Matrices $K_{p}$ and $K_{V}$ elements variation during robot motion. 
Throughout the simulation, all of the physical parameters in the system after 350 seconds time, converge to constant values:

$$
\begin{gathered}
\hat{B}_{1}=0.43, \quad \hat{B}_{2}=0.46, \quad \hat{B}_{3}=1.3 \\
\hat{B}_{4}=0.68, \quad \hat{B}_{5}=0.79, \quad \hat{B}_{6}=1.95, \quad \hat{B}_{7}=3.1 .
\end{gathered}
$$

As an example, the converging of $\hat{B}_{1}, \hat{B}_{4}$ and $\hat{B}_{7}$ parameters are shown in Fig. 9.

\subsection{Control in horizontal path}

As mentioned before, adaptive control includes the two components of feedback and feed forward. Using robot control and revolute joint curves, feedback revolute joint torques can be calculated. A torque diagram is shown in Fig. 10 in four steps. Also, it should be mentioned that matrixes $K_{p}, K_{v}$ are not constant, and are dependent
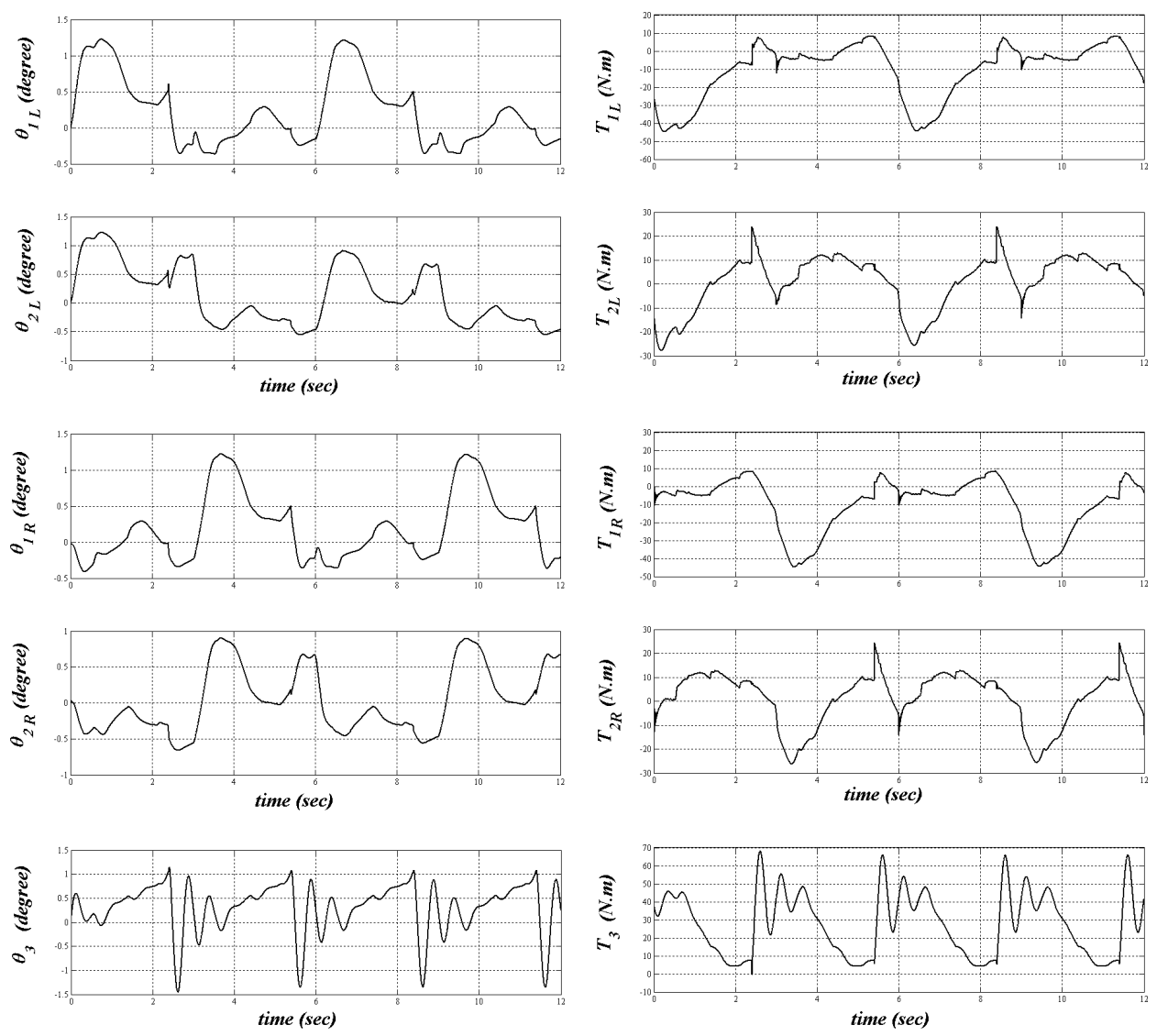

(a) Revolute joint errors.

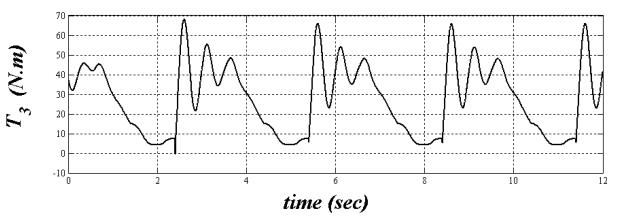

(b) Revolute joint torques.

Fig. 13. Simulation results. 
on the robot's position relations (33) and (34). The variations of these matrices are shown in Fig. 12.

Finally, we use these values and the robot movement (relation 32). The movement of the robot is controlled and the results are shown in Figs. 13(a) and (b), which give the error values for revolute joint and computed torques, respectively.

\section{Simulation}

To check the appropriate operation of the robot and the formulated equations, a 3D model of the robot was created in Visual Nastran Desktop (VND) software (Fig. 11). To make a movement for the created model, the input equations corresponding to each joint were imported into (VND) and the motions of the robot were demonstrated. The stable and harmonic motion of the robot is approved in the computer-aided performance. In addition, the appraisement of the new sole mechanism of the robot verifies the acceptable and correct performance of the mechanism.

\section{Conclusion}

In this paper, a 5 DOF modeling of a bipedal robot is presented. The main advantage of such modeling, which distinguishes it from previous works, is the ability to simulate the motion in a way that is closer to the human walk by using a new toeheel mechanism. After constituting the kinematics equations, the joint's variables for a one-step motion are presented. Also, the dynamical equations corresponding to two stages of motion (single support and double support) based on the ZMP location are derived. For robot equilibrium, a dynamic modification is performed.

As the modification of the torso is defined based on the motion of the legs, computing the curve of the motion of the torso must be done continuously during the motion. The starting angle of the motion of the torso is chosen according to the similarity of the starting point and end point of the motion.

Also, to simulate the motion of the robot and to check the correctness of the results, Visual Nastran Desktop (VND) is used as the dynamic simulator software. The results of simulation verify the correct operation and harmonic motion of the robot.

\section{References}

1. M. Vukobratovic, B. Borovac, D. Surla and D. Stokic, Biped Locomotion: Dynamics, Stability, Control, and Applications (Springer-Verlag, Berlin, 1990).

2. T. McGeer, Passive dynamic walking, Int. J. Robot. Res. 9 (1990) 62-68.

3. J. Yamaguchi, A. Takanashi and I. Kato, Development of a biped walking robot: Compensating for three-axis moment by trunk motion, in IEEE Int. Conf. Intelligent Robotics and Automation, Detroit, USA, 10-15 May (IEEE, 1993), pp. 561-566.

4. K. Hirai, M. Hirose, Y. Haikawa and T. Takenaka, The development of Honda humanoid robot, in IEEE Int. Conf. Robotics and Automation, Leuven, Belgium, 16-20 May (IEEE, 1998), pp. 1321-1326. 
5. F. Najafi, A. Bagheri and R. Farrokhi, Design and motion analysis of a new planar biped, in Climbing and Walking Robots (CLAWAR 2003) Int. Conf., Catania, Italy (2003), pp. 551-557.

6. R. Farrokhi, The control of a walking robot in upward-downward movement applying ZMP, Master degree thesis, Guilan University (2003).

7. K. Mitobe, G. Capi and Y. Nasu, Control of walking robots based on manipulation of the zero moment point, Robotica 18(6) (2000) 651-657.

8. G. Tan and P. Zhang, A new method for key dynamic parameters of dynamic walking of bipedal robots, in Proc. IEEE Int. Conf. Intelligent Processing Systems, Beijing, China, 28-31 October (IEEE, 1998), pp. 1292-1296.

9. C.-L. Shih, Analysis of the dynamics of a biped robot with seven degrees of freedom, in IEEE Int. Conf. Robotics and Automation, Minneapolis, USA, 22-28 April (IEEE, 1996), pp. 3008-3013.

10. J. Pratt, Exploiting inherent robustness and natural dynamics in the control of bipedal walking robots, Ph.D. thesis (MIT, 2000).

11. J. Pratt, Ch. Chew, A. Torres, P. Dilworth and G. Pratt, Virtual model control: An intuitive approach for bipedal locomotion, Int. J. Robot. Res. 20 (2001) 176.

12. J. Slotine and W. Li, Applied Nonlinear Control (Prentice-Hall, Cambridge, 1991).

13. F. Najafi, A. Bagheri and R. Farrokhi, Adaptive control algorithm for single support phase of motion of a new biped robot, in 9th IEEE Int. Conf. Methods and Models in Automation and Robotics (MMAR 2003), Poland (2003), pp. 1093-1100.

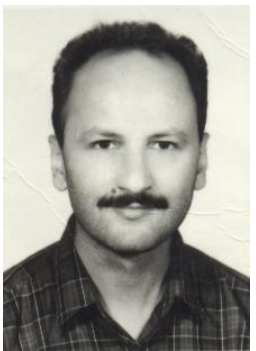

Ahmad Bagheri was born in Tehran, Iran, 1966. He received his B.Sc. degree in Mechanical Engineering from the University of Mashhad in 1989, his M.Sc. degree from the University of Tarbiat Modares in 1993, and his Ph.D. degree in Mechatronics at CTU Prague in 1997. He worked for four years for the Iranian Ministry of Industries. He is currently serving as an Assistant Professor and the Head of the Mechanical Engineering Department, Guilan University. He has published 48 papers in conferences proceedings and journals. His research interests include biped locomotion, robot modeling and control, industrial robotics and mechatronics.

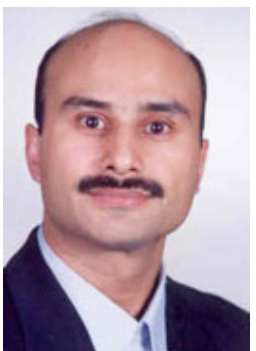

Farid Najafi was born in Tehran, Iran, 1967. He received his B.Sc. degree in Mechanical Engineering from Sharif University of Technology in 1990 and his M.Sc. and Ph.D. degrees in Robotic Systems from Moscow State Technical University in 1993 and 1996, respectively. He served as Director of the actuators research laboratory, Head of the Department of Mechanics (solid design), Deputy Research Affairs of the Mechanical Engineering Faculty at K. N. Toosi University of Technology and Assistant Professor of K.N.T.U. of Technology. His research interests include dynamic systems, control of robots, walking robots and mechatronics.

Reza Farrokhi was born in Tehran, Iran, 1978. He received his M.Sc. degree in Mechanical Engineering from Guilan University in 2003. 


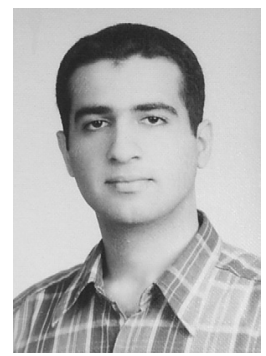

Rahman Yousefi Moghaddam was born in Rasht, Iran in 1980. He received his B.Sc. and M.Sc degrees in Mechanical Engineering from Guilan University in 2002 and 2005, respectively. He is now doing a Ph.D. program in Mechanical Engineering at the University of Manitoba, Canada. His research interests include robotics and the control of the robots, mechatronics, and manufacturing systems.

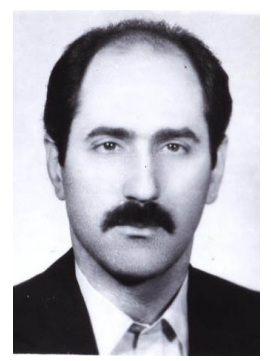

Mohammad Ebrahim Felezi was born in Rasht, Iran in 1957. He received his B.Sc. and M.Sc. degrees in Mechanical Engineering from Tehran University in 1985 and 1988, respectively. He is now studying for his Ph.D degree in the Mechanical Engineering Department at Guilan University. He worked for five years for the Iranian Ministry of Industries. He is also a member of Civil Engineering Organization. He is also a lecturer in the Mechanical Engineering Department at Guilan University. He has published three journal papers and a textbook about turbomachinary. He has supervised different B.Sc. projects in the above-mentioned field. His main research interest is in mechanism design. 